\title{
SELF MOTIONS OF A SPECIAL 3-RPR PLANAR PARALLEL ROBOT
}

\author{
Damien Chablat, Phlippe Wenger \\ Institut de Recherche en Communications et Cybernétique de Nantes \\ 1, rue de la Noë, 44321 Nantes, FRANCE \\ [damien.chablat, philippe.wenger] @irccyn.ec-nantes.fr
}

Ilian A. Bonev

École de technologie supérieure

1100, rue Notre-Dame Ouest, Montréal (Québec) H3C 1K3, CANADA

ilian.bonev@etsmtl.ca

\begin{abstract}
This paper studies the kinematic geometry of a $3-\underline{R} P R$ planar parallel robot with actuated base joints and congruent equilateral base and mobile platform. The singularity loci are first determined. Then the global behavior at all singularities is geometrically described by studying the degeneracies of the direct kinematic model. This study is made easier by the fact that one of the two direct kinematic solutions is trivial and singular. It is shown that this parallel robot has Cardanic self motions.
\end{abstract}

Keywords: Kinematic geometry, planar parallel mechanism, singularity, self motion

\section{Introduction}

Often, the main drawback of choosing design conditions for which the direct kinematic problem of a parallel robot becomes simplified is the appearance of self motions (Husty and Zsombor-Murray, 1994; Karger, 2002; Wohlhart, 2002). While self motions are certainly the worst type of singularity a parallel robot could cross, they should simply be avoided like any other type of singularity. Then, why not use parallel robots with self motions if these robots have simpler direct kinematics?

For example, in a recent paper (Bonev et al., 2006), the authors have shown that all singularities of the popular spherical parallel robot design, known as the Agile Eye, correspond to self motions, yet this design is arguably the "best" spherical wrist. (In the Agile Eye, any two axes that are fixed to one another are orthogonal.) Indeed, the singularity loci of the Agile Eye are not surfaces but curves, which means that the robot's theoretical workspace is undivided and unlimited. Furthermore, 
while the Agile Eye still has eight assembly modes, like any other $3-\underline{R} R R$ parallel wrist ( $R$ stands for revolute and $P$ for prismatic joint, and an underline indicates which joint is actuated), half of them correspond to four singular orientations and the other half are easily found in cascade.

Self motions have been investigated mainly for Stewart-Gough platforms. Few planar parallel robots with self motions have been studied, and the kinematics of the general $3-\underline{R} P R$ planar parallel robot was only briefly considered in two papers (Merlet, 1996; Bonev et al., 2003). We came up with a special design for this peculiar planar parallel robot on a fruitless search for a planar equivalent to the Agile Eye. While our special 3- $R P R$ planar parallel robot is certainly not the most useful one, the study of its self motions brings insight into the complex relationship between direct kinematics and singularities of parallel robots.

In the next section, we will briefly present the analytic expression for the singularity loci of our special $3-\underline{R} P R$ planar parallel robot. We will identify a range of orientations for which the robot has a sufficiently large singularity-free workspace. Then, in Section 3, we will describe the several types of singular configurations by studying the degeneracies of the direct kinematics and show that they belong to self motions. Conclusions are given in the last section.

\section{Singularity Loci}

The special 3- $\underline{R} P R$ planar parallel robot is shown in Fig. 1. Its mobile platform and base form congruent equilateral triangles. We denote with $O_{i}$ and $B_{i}$ (in this paper, $i=1,2,3$ ) the intersections of the base and platform revolute joint axes, respectively, with a plane normal to these axes. Then, let $O x y$ and $C x^{\prime} y^{\prime}$ be the base and mobile reference frames, respectively. The generalized coordinates locating the mobile platform, i.e., the mobile frame $C x^{\prime} y^{\prime}$, in the base frame $O x y$ will be denoted by $x, y$, and $\phi$. We define each active-joint variable $\theta_{i}$ as the angle between the $x$-axis and a unit vector $\mathbf{v}_{i}$ that defines the direction of the prismatic joint of leg $i$, measured in counter-clockwise sense. Finally, the directed distance from point $O_{i}$ to point $B_{i}$ along vector $\mathbf{v}_{i}$ is denoted by $\rho_{i}$.

Skipping the derivation and referring the reader to (Bonev et al., $2003)$, the velocity equation for the $3-\underline{R} P R$ planar parallel robot is

$$
\mathbf{A} \dot{\boldsymbol{q}}=\left[\begin{array}{cc}
m_{1} & \mathbf{f}_{1}^{T} \\
m_{2} & \mathbf{f}_{2}^{T} \\
m_{3} & \mathbf{f}_{3}^{T}
\end{array}\right]\left[\begin{array}{c}
\dot{\phi} \\
\dot{x} \\
\dot{y}
\end{array}\right]=\left[\begin{array}{ccc}
\rho_{1} & 0 & 0 \\
0 & \rho_{2} & 0 \\
0 & 0 & \rho_{3}
\end{array}\right]\left[\begin{array}{c}
\dot{\theta_{1}} \\
\dot{\theta_{2}} \\
\dot{\theta_{3}}
\end{array}\right]=\mathbf{B} \dot{\boldsymbol{\theta}}
$$

where

$$
\mathbf{f}_{i}=\mathbf{E} \mathbf{v}_{i}=\left[\begin{array}{c}
-\sin \theta_{i} \\
\cos \theta_{i}
\end{array}\right]=\mathbf{E}\left(\mathbf{r}_{O C}+\mathbf{r}_{C B_{i}}-\mathbf{r}_{O O_{i}}\right) / \rho_{i}
$$




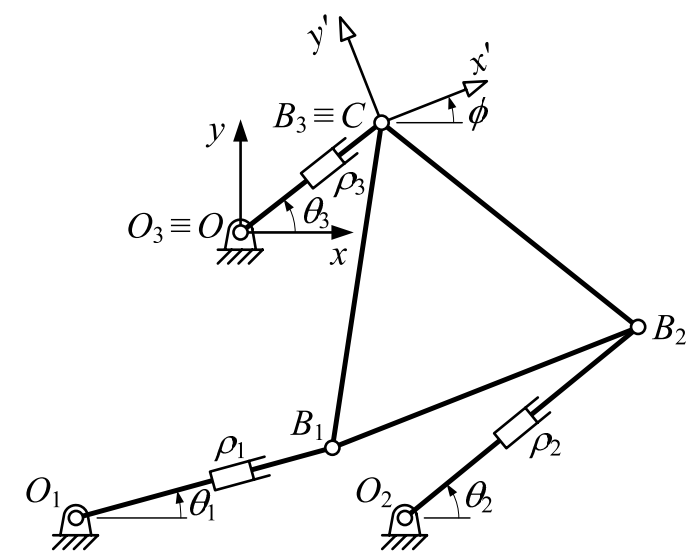

Figure 1. 3- $\underline{R} P R$ planar parallel robot with congruent equilateral base and platform.

$$
m_{i}=\mathbf{f}_{i}^{T} \mathbf{E} \mathbf{r}_{C B_{i}}=\mathbf{f}_{i}^{T} \mathbf{E} \mathbf{R} \mathbf{r}_{C B_{i}}^{\prime}
$$

and

$$
\mathbf{E}=\left[\begin{array}{cc}
0 & -1 \\
1 & 0
\end{array}\right], \mathbf{R}=\left[\begin{array}{cc}
\cos \phi & -\sin \phi \\
\sin \phi & \cos \phi
\end{array}\right]
$$

Furthermore, $\mathbf{r}_{O C}=[x, y]^{T}, \mathbf{r}_{O O_{i}}$ and $\mathbf{r}_{C B_{i}}$ are the vectors along $O O_{i}$ and $C B_{i}$, respectively, expressed in the base frame, and $\mathbf{r}_{C B_{i}}^{\prime}$ is the vector along $C B_{i}$ expressed in the mobile frame. Without loss of generality, we define these vectors as

$$
\begin{gathered}
\mathbf{r}_{O O_{1}}=\mathbf{r}_{C B_{1}}^{\prime}=\left[\begin{array}{c}
-1 / 2 \\
-\sqrt{3} / 2
\end{array}\right], \mathbf{r}_{O O_{2}}=\mathbf{r}_{C B_{2}}^{\prime}=\left[\begin{array}{c}
1 / 2 \\
-\sqrt{3} / 2
\end{array}\right] \\
\mathbf{r}_{O O_{3}}=\mathbf{r}_{C B_{3}}^{\prime}=\left[\begin{array}{l}
0 \\
0
\end{array}\right]
\end{gathered}
$$

or, in other words, we set the sides of the base and platform to unity, and fix the base and platform frames at points $O_{3}$ and $B_{3}$, respectively.

Type 2 singularities occur when $\mathbf{A}$ is singular. Geometrically, this means that the lines normal to the directions of the prismatic joints and passing through points $B_{i}$ are concurrent or parallel. Simplifying the determinant of $\mathbf{A}$ and zeroing yields:

$$
\operatorname{det}(\mathbf{A})=\frac{\sqrt{3}(\cos \phi-1)}{2 \rho_{1} \rho_{2} \rho_{3}}\left(\left(x-x_{c}\right)^{2}+\left(y-y_{c}\right)-r^{2}\right)=0
$$

where

$$
x_{c}=-\frac{1}{\sqrt{3}} \sin \phi, y_{c}=-\frac{1}{\sqrt{3}}(1-\cos \phi), r=\sqrt{\frac{2(1-\cos \phi)}{3}} .
$$




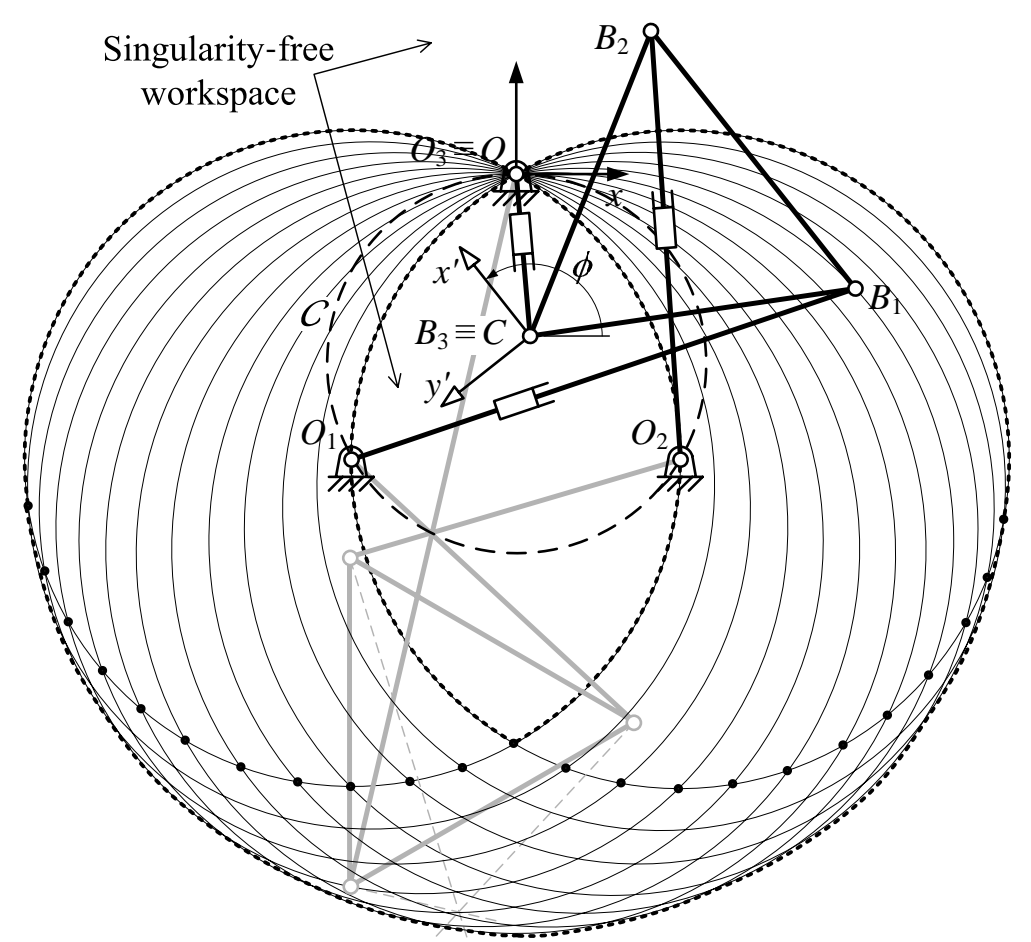

Figure 2. Singularity loci and singularity-free workspace for $\phi \in\left[120^{\circ}, 240^{\circ}\right]$.

Therefore, Type 2 singularity loci for this mechanism are (a) the whole $x y$-plane, when $\phi=0$ (the directions of all prismatic joints are parallel), and (b) a circle of radius $r$, centered at $\left(x_{c}, y_{c}\right)$, which passes through $\mathrm{O}_{3}$, when $\phi \neq 0$. Note, however, that Eq. (1) is of indeterminate form $(0 / 0)$ at a Type 1 singularity. Indeed, at a Type 1 singularity, a motor can freely rotate without affecting the pose of the platform. Such a pose would be Type 2 singular only for specific combinations of the activejoint variables that correspond to singular legs.

We will conclude this section by proposing a range of orientations for which the singularity-free workspace is sufficiently large. Apparently, this range needs to exclude $\phi=0$, and if symmetry is to be observed, the best choice would be a range centered at $\phi=180^{\circ}$. Figure 2 shows our parallel robot with a series of Type 2 singularity circles corresponding to $\phi=120^{\circ}+k 10^{\circ}(k=0,1,2, \ldots, 12)$. The dots correspond to Type 1 singularities. The singularity-free workspace for the orientation range $\phi \in\left[120^{\circ}, 240^{\circ}\right]$ is the one that excludes the circle-swept region in Fig. 2.

Based on this purely algebraic analysis, it is certainly not obvious, but one can verify by using Eq. (5) that for any Type 2 singular configuration for which $\phi \neq 0$, lines $O_{i} B_{i}$ intersect at a common point lying on the circumcircle of the base (see the gray-colored configuration in Fig. 2). 


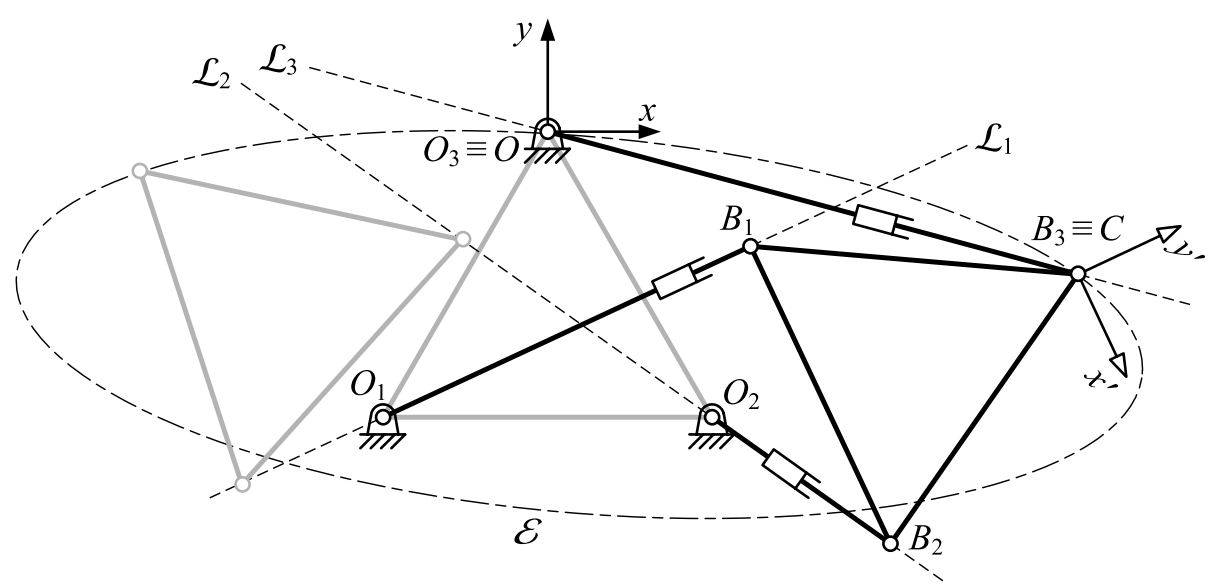

Figure 3. Geometric interpretation of the direct kinematic model.

\section{Degeneracies of the Direct Kinematics}

In order to study the global behavior of the parallel robot at all singularities, we will analyze its direct kinematic model. Indeed, this approach is the most intuitive one and gives a clear geometric interpretation of all singular configurations. It is usually very difficult or even impossible to follow this approach, but in our case the direct kinematic model is particularly simple. Indeed, whatever the active-joint variables, there is always the trivial solution when the base and platform coincide.

Merlet (1996) showed that the direct kinematic problem of $3-\underline{R} P R$ planar parallel robots is equivalent to intersecting an ellipse with a line, but did not study any special cases. Let us dismount the revolute joint at $B_{3}$. For given active-joint variables, points $B_{1}$ and $B_{2}$ are constrained to move along two lines (Fig. 3), $\mathcal{L}_{1}$ and $\mathcal{L}_{2}$, respectively, and the mobile platform undergoes a Cardanic movement (see for example Tischler et al., 1998). As a result, $B_{3}$ describes a curve, $\mathcal{E}$, that is an ellipse, two parallel lines, a line, or a doubly traced line segment. Obviously, this curve contains point $O_{3}$ (i.e., $O$ ). Considering leg $3, B_{3}$ should also lie on a line passing through $O_{3}$, denoted by $\mathcal{L}_{3}$. Thus, the direct kinematic problem is equivalent to finding the intersections between $\mathcal{L}_{3}$ and $\mathcal{E}$.

Let us now derive the elliptic curve $\mathcal{E}$. We can write the following closure equation for point $C \equiv B_{3}$ :

$$
\mathbf{r}_{O C}=\mathbf{r}_{O O_{1}}+\rho_{1} \mathbf{v}_{1}-\mathbf{r}_{C B_{1}}=\mathbf{r}_{O O_{2}}+\rho_{2} \mathbf{v}_{2}-\mathbf{r}_{C B_{2}} .
$$

This yields the following system of two equations:

$$
\begin{aligned}
-1+\rho_{1} \cos \theta_{1}+\cos \phi-\rho_{2} \cos \theta_{2} & =0, \\
\rho_{1} \sin \theta_{1}+\sin \phi-\rho_{2} \sin \theta_{2} & =0 .
\end{aligned}
$$




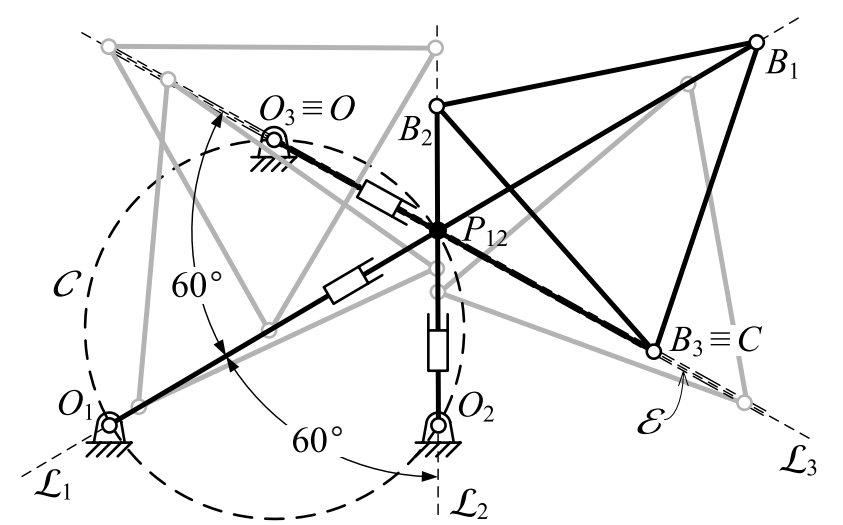

Figure 4. Cardanic self motion when the legs are concurrent and make equal angles.

Solving Eqs. (7) and (8) for $\rho_{1}$ and $\rho_{2}$, and substituting the results in Eq. (6), we obtain the following parametric equation in $\phi$ :

$$
\begin{aligned}
& x=\left(\frac{\cos \theta_{1} \sin \theta_{2}}{\sin \left(\theta_{1}-\theta_{2}\right)}+\frac{1}{2}\right)(\cos \phi-1)-\left(\frac{\cos \theta_{1} \cos \theta_{2}}{\sin \left(\theta_{1}-\theta_{2}\right)}+\frac{\sqrt{3}}{2}\right) \sin \phi, \\
& y=\left(\frac{\sin \theta_{1} \sin \theta_{2}}{\sin \left(\theta_{1}-\theta_{2}\right)}+\frac{\sqrt{3}}{2}\right)(\cos \phi-1)-\left(\frac{\sin \theta_{1} \cos \theta_{2}}{\sin \left(\theta_{1}-\theta_{2}\right)}-\frac{1}{2}\right) \sin \phi .
\end{aligned}
$$

The above parametric equation is not defined when $\sin \left(\theta_{1}-\theta_{2}\right)=0$, a case that will be studied later. If $\sin \left(\theta_{1}-\theta_{2}\right) \neq 0$, i.e., if $\mathcal{L}_{1}$ and $\mathcal{L}_{2}$ are not parallel, then the elliptic curve degenerates if, and only if, its curvature is zero for any $\phi$. The curvature, $\kappa$, of $\mathcal{E}$ can be derived as

$$
\kappa=\frac{\dot{x} \ddot{y}-\dot{y} \ddot{x}}{\left(\dot{x}^{2}+\dot{y}^{2}\right)^{3 / 2}}=\frac{\sin \left(\theta_{1}-\theta_{2}+\pi / 3\right)}{D^{3}},
$$

where $D$ is a relatively large expression in $\theta_{1}, \theta_{2}$, and $\phi$.

Therefore, when $\mathcal{L}_{1}$ and $\mathcal{L}_{2}$ make a $60^{\circ}$ angle, $\kappa=0$, and $\mathcal{E}$ degenerates to a line. In other words, if, and only if, the point of intersection between $\mathcal{L}_{1}$ and $\mathcal{L}_{2}$, denoted by $P_{12}$, lies on the curcumcircle of the base, denoted by $\mathcal{C}, \mathcal{E}$ degenerates to a line (Fig. 4 ). In fact, it degenerates to a doubly traced line segment of length $4 / \sqrt{3}$ (Tischler et al., 1998). This line segment is centered at $P_{12}$ and passes through $O$. When $P_{12} \equiv O$, the doubly traced line segment is parallel to $\mathrm{O}_{1} \mathrm{O}_{2}$.

As we said before, the direct kinematic problem is equivalent to finding the two intersection points between $\mathcal{L}_{3}$ and $\mathcal{E}$, of which one is always $O$. We will not present an actual algorithm for determining the other intersection point $(x, y)$ and the corresponding platform orientation $\phi$, but only investigate the singular configurations corresponding to all particular cases in which there is a single or infinitely many solutions. 
Case 1a: $\sin \left(\theta_{1}-\theta_{2}\right)=0$ and $\cos \theta_{1} \cos \theta_{2} \neq 0$

In this case, $\mathcal{L}_{1}$ and $\mathcal{L}_{2}$ are parallel and $\mathcal{E}$ degenerates to two lines parallel to $\mathcal{L}_{1}$ and $\mathcal{L}_{2}$, one of which passes through $O$.

$x$ If $\mathcal{L}_{3}$ is parallel to $\mathcal{L}_{1}$ and $\mathcal{L}_{2}$, the platform vertices can slide along $\mathcal{L}_{1}, \mathcal{L}_{2}$ and $\mathcal{L}_{3}$, with $\phi=0$, even though all actuators are blocked.

$\boldsymbol{x}$ If $\mathcal{L}_{3}$ is not parallel to $\mathcal{L}_{1}$ and $\mathcal{L}_{2}$, the platform can assume a nonsingular configuration, as well as the trivial solution $\mathbf{q}=\mathbf{0}$, for which the corresponding configuration is only Type 1 singular (i.e., the platform is not shaky; in fact, it is jammed).

Case 1b: $\cos \theta_{1}=0$ and $\cos \theta_{2}=0$

In this case, $\mathcal{L}_{1}$ and $\mathcal{L}_{2}$ are parallel to the $y$-axis and $\mathcal{E}$ degenerates to a single line parallel to $\mathcal{L}_{1}$ and $\mathcal{L}_{2}$, and passing through $O$.

$x$ If $\mathcal{L}_{3}$ is parallel to $\mathcal{L}_{1}$ and $\mathcal{L}_{2}$, the platform vertices can slide along $\mathcal{L}_{1}, \mathcal{L}_{2}$ and $\mathcal{L}_{3}$, with $\phi=0$, even though all actuators are blocked.

$\boldsymbol{x}$ If $\mathcal{L}_{3}$ is not parallel to $\mathcal{L}_{1}$ and $\mathcal{L}_{2}$, the platform can assume only the trivial solution $\mathbf{q}=\mathbf{0}$, and the configuration is Type 1 and Type 2 singular (the platform can rotate infinitesimally).

Case 2: $\sin \left(\theta_{1}-\theta_{2}+\pi / 3\right)=0$

In this case, $\mathcal{L}_{1}$ and $\mathcal{L}_{2}$ make a $60^{\circ}$ angle and their intersection point, $P_{12}$, lies on $\mathcal{C}$ (Fig. 4). The curve $\mathcal{E}$ degenerates to a doubly traced line segment passing through $P_{12}$ and $O$ (if $P_{12} \equiv O, \mathcal{E}$ is parallel to $O_{1} O_{2}$ ).

$\boldsymbol{x}$ If $\mathcal{L}_{3}$ is collinear with $\mathcal{E}$, then point $B_{3}$ can slide along $\mathcal{E}$ while the platform changes orientation simultaneously (Fig. 4), even though all actuators are blocked (as in Reuleaux straight-line mechanism).

$\boldsymbol{x}$ If $\mathcal{L}_{3}$ is not collinear with $\mathcal{E}$, then the platform can assume two possible poses. The first one is the trivial solution $\mathbf{q}=\mathbf{0}$, while the second one is $\mathbf{q}=\left[180^{\circ}, 0,0\right]^{T}$. For both poses, the corresponding configurations are only Type 1 singular (the platform is jammed).

Case 3: $\sin \left(\theta_{1}-\theta_{2}+\pi / 3\right) \sin \left(\theta_{1}-\theta_{2}\right) \neq 0$

In this case, $\mathcal{L}_{1}$ and $\mathcal{L}_{2}$ intersect at a point that does not lie on $\mathcal{C}$, and $\mathcal{E}$ is an ellipse (Fig. 3).

$\boldsymbol{x}$ If $\mathcal{L}_{3}$ is tangent to $\mathcal{E}$ at $O$, then the platform can assume only the pose $\mathbf{q}=\mathbf{0}$, and the corresponding configuration is both Type 1 and Type 2 singular (there is no self motion). Indeed, it can be shown that at this configuration, the normals to $\mathcal{L}_{1}, \mathcal{L}_{2}$ and $\mathcal{L}_{3}$ passing through $B_{1}, B_{2}$ and $B_{3}$, respectively, are concurrent.

$\boldsymbol{x}$ If $\mathcal{L}_{3}$ is not tangent to the ellipse, then the mobile platform can assume the pose $\mathbf{q}=\mathbf{0}$, for which the corresponding configuration is only Type 1 singular, and another nonsingular pose (Fig. 3). 


\section{Conclusions}

This paper described the kinematic geometry of a $3-\underline{R} P R$ planar parallel robot with congruent equilateral base and mobile platform. It was shown that the only singular configurations, for which the base and mobile platform do not coincide, belong to self motions. They occur when the legs intersect at the circumcircle of the base or are parallel. Once again, It was demonstrated that careful analysis of the degeneracies of the direct kinematic model provides a convenient geometrical tool for better understanding the global behavior of singularities.

Parallel robots with identical base and mobile platform allow self motions but have interesting characteristics and simpler geometric models. The authors believe that these robots deserve further attention as they are likely to find use in practice or lead to better understanding some theoretical issues. This design simplification approach will next be applied to $3-\underline{R} R R$ planar parallel robots. These robots are undoubtedly the cheapest alternative for planar motion, from the hardware point of view, but are still too complex to design optimally.

\section{References}

Bonev, I.A., Chablat, D., and Wenger, P. (2006), Working and assembly modes of the Agile Eye, Proceedings of the 2006 IEEE International Conference on Robotics and Automation, Orlando, Florida, May 15-19.

Bonev, I.A., Zlatanov, D., and Gosselin, C.M. (2003), Singularity analysis of 3-DOF planar parallel mechanisms via screw theory, Journal of Mechanical Design, vol. 125, no. 3 , pp. $573-581$.

Husty, M., and Zsombor-Murray, P. (1994), A special type of singular Stewart-Gough platform, Advances in Robot Kinematics and Computational Geometry, J. Lenarčič; and B. Ravani (eds.), Kluwer Academic Publishers, The Netherlands, pp. 449-458.

Karger, A. (2002), Singularities and self motions of a special type of platforms, $A d-$ vances in Robot Kinematics, J. Lenarčič; and F. Thomas (eds.), Kluwer Academic Publishers, The Netherlands, pp. 449-458.

Merlet, J.-P. (1996), Direct kinematics of planar parallel manipulators, Proceedings of the 1996 IEEE International Conference on Robotics and Automation, Minneapolis, Minnesota, pp. 3744-3749.

Tischler, C.R., Hunt, K.H., and Samuel, A.E. (1998), A spatial extension of Cardanic movement: its geometry and some derived mechanisms, Mechanism and Machine Theory, vol. 33, no. 8, pp. 1249-1276.

Wohlhart, K. (2002). Synthesis of architecturally mobile double-planar platforms, Advances in Robot Kinematics, J. Lenarčič; and F. Thomas (eds.), Kluwer Academic Publishers, The Netherlands, pp. 473-482. 